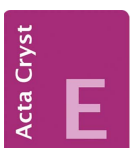

CRYSTALLOGRAPHIC COMMUNICATIONS

ISSN 2056-9890

Received 4 October 2020

Accepted 17 November 2020

Edited by W. T. A. Harrison, University of Aberdeen, Scotland

Keywords: co-crystal structure; amine carboxyborane; acetaminophen; CORCB.

CCDC reference: 1828957

Supporting information: this article has supporting information at journals.iucr.org/e

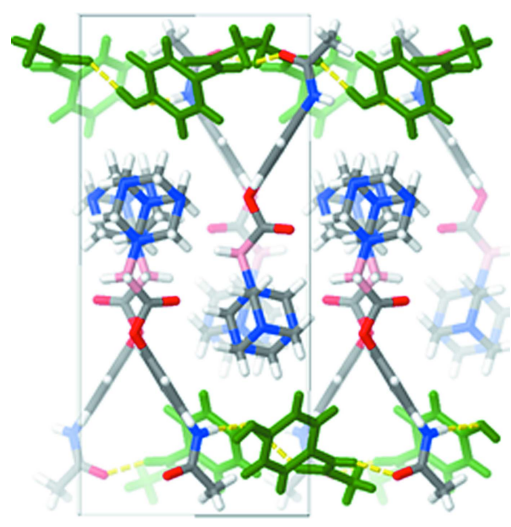

\section{Synthesis and structure of a 1:1 co-crystal of hexamethylenetetramine carboxyborane and acetaminophen}

\author{
Theppawut Ayudhya, ${ }^{\mathrm{a}}$ Casey Raymond ${ }^{\mathrm{b}}$ and Nin Dingra ${ }^{\mathrm{a} *}$
}

${ }^{\mathbf{a}}$ Department of Chemistry, University of Texas Permian Basin, Odessa, Texas, USA, and ${ }^{\mathbf{b}}$ Department of Chemistry, State University of New York at Oswego, Oswego, New York, USA. *Correspondence e-mail: dingra_n@utpb.edu

Hexamethylenetetramine carboacetaminophenborane, a molecule with two pharmacophores attached to a central carboxyborate moiety, was synthesized and crystals were grown with an acetaminophen co-crystal former to result in the title 1:1 co-crystal [hexamethylenetetramine 4-acetamidophenyl 2-boranylacetate-4-acetamidophenol (1/1)], $\mathrm{C}_{15} \mathrm{H}_{22} \mathrm{BN}_{5} \mathrm{O}_{3} \cdot \mathrm{C}_{8} \mathrm{H}_{9} \mathrm{NO}_{2}$. In the first of these molecules, both the borate-ester and acetylamino groups are considerably twisted away from the plane of the intervening benzene ring [dihedral angles = 76.89 (9) and $65.42(9)^{\circ}$, respectively]. The extended structure of this co-crystal features $\mathrm{N}-\mathrm{H} \cdots \mathrm{O}$ and $\mathrm{O}-\mathrm{H} \cdots \mathrm{O}$ hydrogen bonds, which link the components into (100) sheets and weak $\mathrm{C}-\mathrm{H}$... O hydrogen bonds help to consolidate the structure.

\section{Chemical context}

Crystal structures of pure drugs are of great interest in the pharmaceutical industry since these structures provide an understanding of the intermolecular interactions that explain the physical and chemical properties of the solid (Desiraju, 2007). Modifications made to the active pharmaceutical ingredients to enhance the biological availability often include crystal engineering. For instance, the recrystallization of acetaminophen, $\mathrm{C}_{8} \mathrm{H}_{9} \mathrm{NO}_{2}$ (also known as paracetamol), gives crystal form II, which displays better solubility and compressibility than form I (Naumov et al., 1998; Agnew et al., 2016). Another approach that has been brought into attention is using crystal formers or co-formers to improve the physicochemical characteristics of the solids. Recent developments in co-crystallization show potential advantages of drug-coformer co-crystals as well as drug-drug co-crystals (Kaur et al., 2017; Cheney et al., 2011; Nugrahani et al., 2007; Dalpiaz et al., 2018).

A group of organo-boron compounds, namely amine carboxyboranes, have been studied extensively for their diverse biological effects such as anti-inflammatory, antineoplastic and anti-osteoporotic activities (Hall et al., 1995, 1990; Murphy et al., 1996). Their fundamental structure contains tetravalent amines connected to a boron atom of the carboxyborane moiety with an $\mathrm{N}-\mathrm{B}$ coordinate covalent bond (Spielvogel et al., 1976). As a result of the ease of structural transformability, this group is very amenable to modification such as exchanging various amine groups and esterification on the carboxyborate. Our interest in amine carboxyboranes stemmed from their innate structure that undergoes decarbonylation to produce $\mathrm{CO}, \mathrm{H}_{2}$, and the amine group when placed in aqueous solution. We have shown amine 
carboxyboranes to be a group of molecules that can be used as carbon monoxide releasers (Ayudhya et al., 2017). Moreover, we have recently reported that this process is accelerated by reactive oxygen species (ROS) increasing the rate at which $\mathrm{CO}$ and the amine group is released (Ayudhya et al., 2018). Considering the amine compounds are drug molecules, carboxyboranes can be used as a system to deliver drugs that contain amino groups. Since we started our endeavor with drug-conjugated carboxyboranes (Ayudhya et al., 2018), we speculated that carboxyboranes may be able to carry more than one drug. In addition to the amine group on the boron atom, ester and amide derivatives at the carboxyborate end have been shown previously (Das et al., 1990).

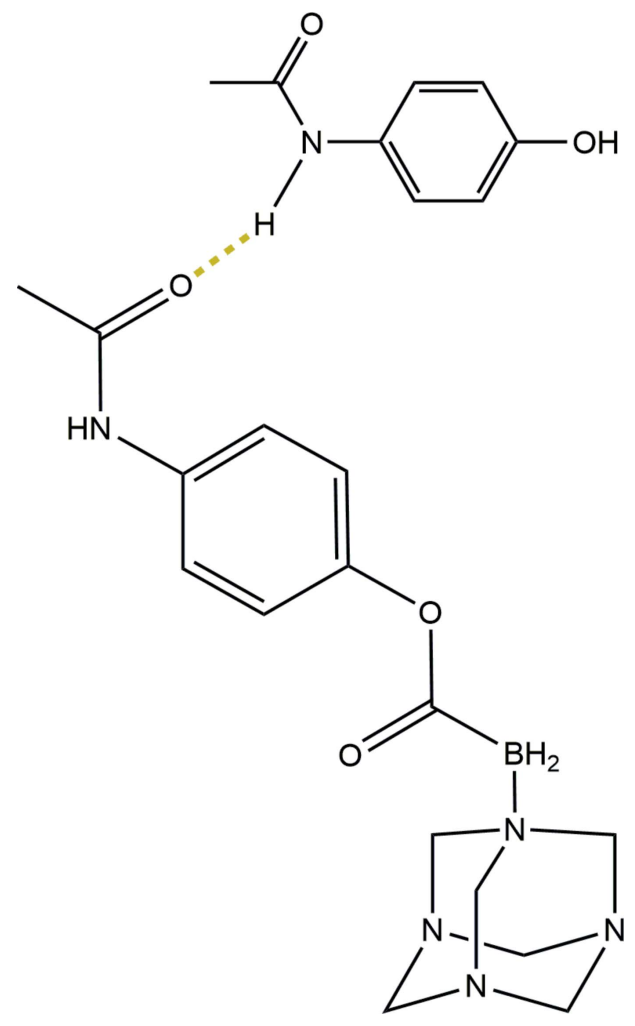

As part of this work, we now describe the crystal structure of the title co-crystal, $\mathrm{C}_{15} \mathrm{H}_{22} \mathrm{BN}_{5} \mathrm{O}_{3} \cdot \mathrm{C}_{8} \mathrm{H}_{9} \mathrm{NO}_{2}$, (I), which resulted from the synthetic concept that conjugating two different pharmacophores to the carboxyborate moiety may make a molecule that has multiple biological effects.

\section{Structural commentary}

The asymmetric unit of the resulting monoclinic crystal (space group $P 2_{1} / c$ ) contains one $\mathrm{C}_{15} \mathrm{H}_{22} \mathrm{BN}_{5} \mathrm{O}_{3}$ ester (CORCB-1APAP) and one $\mathrm{C}_{8} \mathrm{H}_{9} \mathrm{NO}_{2}$ acetaminophen molecule (Fig. 1). The hexamethylenetetraamine (hmta) moiety of the ester is syn to the $\mathrm{C} 9=\mathrm{O} 3$ carboxy carbonyl group and the aromatic C3-C8 ring is approximately perpendicular to the plane of the $\mathrm{B} 1 / \mathrm{C} 9 / \mathrm{O} 2 / \mathrm{O} 3$ ester carboxylate group [dihedral angle = $\left.76.89(9)^{\circ}\right]$ while the $\mathrm{C} 1 / \mathrm{C} 2 / \mathrm{N} 1 / \mathrm{O} 1$ acetylamino group is twisted out of plane of the ring by $65.42(9)^{\circ}$; the dihedral angle between the pendant groups is $11.70(10)^{\circ}$.
Based on the observed geometry, we may assume that the bonding in this difunctionalized carboxyborate is very similar to that in the previously reported crystal structure of $\mathrm{C}_{7} \mathrm{H}_{15} \mathrm{BN}_{4} \mathrm{O}_{2}$ or CORCB-1 [Ayudhya et al., 2017; Cambridge Structural Database (Groom et al., 2016) refcode UDAQOI]. The only significant difference is in the slightly longer C9-O2 single bond, 1.399 (2) $\AA$ in the difunctionalized title compound compared to 1.353 (3) $\AA$ in CORCB-1. This lengthening is expected to be due to the weak ester bond, which is confirmed by rapid hydrolysis. There are only small differences in $\mathrm{B}-\mathrm{N}$ and $\mathrm{B}-\mathrm{C}$ bond lengths between the two materials with some lengthening seen in the difunctionalized compound. In the co-crystallized acetaminophen molecule in (I), the dihedral angle between the $\mathrm{C} 18-\mathrm{C} 23$ benzene ring and the acetylamino $\mathrm{C} 16 / \mathrm{C} 17 / \mathrm{N} 6 / \mathrm{O} 5$ grouping is $54.61(10)^{\circ}$.

\section{Supramolecular features}

During crystallization, the new difunctionalized molecule, CORCB-1-APAP, forms a co-crystal with acetaminophen at a 1:1 ratio with hydrogen-bonding interactions (Table 1) between them (Figs. 2 and 3). In comparison to the CORCB-1 crystal reported previously, which features hydrogen bonds between the amino and carboxylic acid groups (Ayudhya et al., 2017), this new structure cannot form hydrogen bonds in the

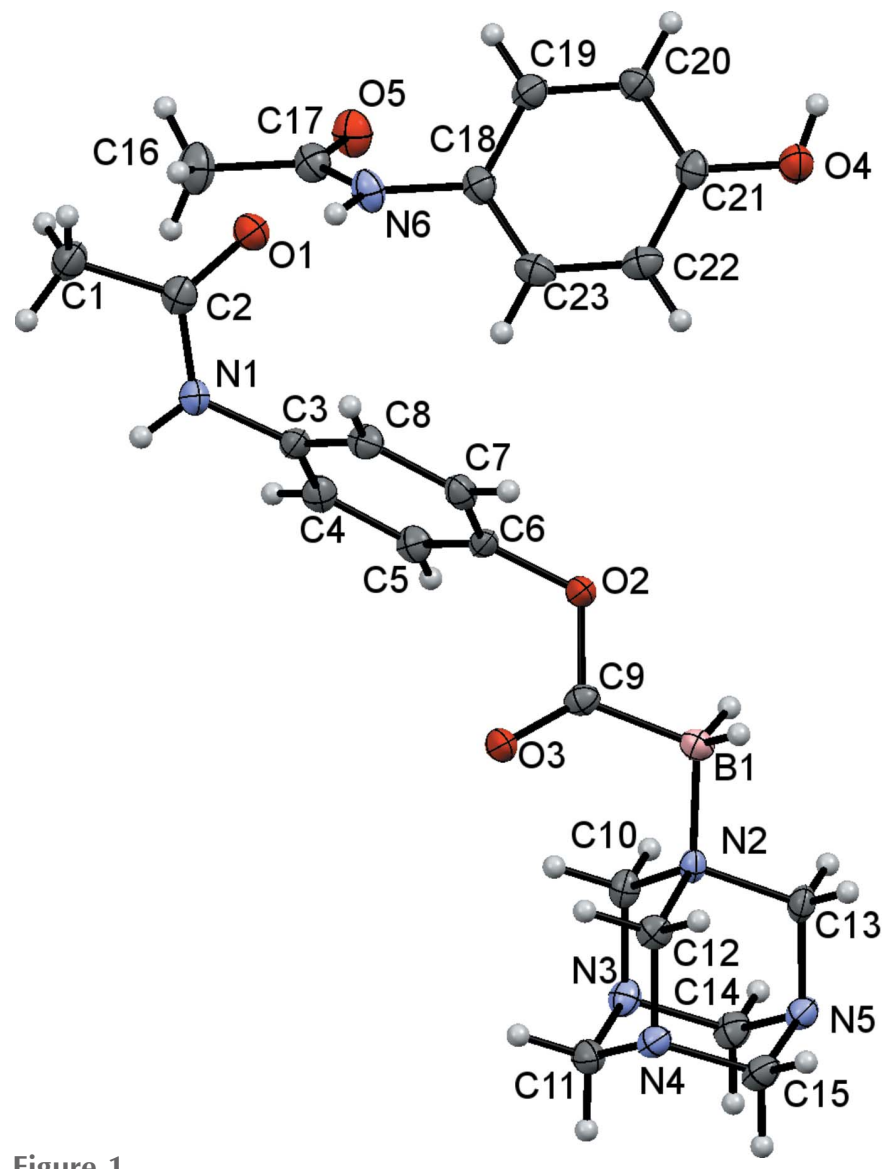

Figure 1

The molecular structure of (I) with displacement ellipsoids drawn at the $50 \%$ probability level. 
Table 1

Hydrogen-bond geometry $\left(\AA,^{\circ}\right)$.

\begin{tabular}{|c|c|c|c|c|}
\hline$D-\mathrm{H} \cdots A$ & $D-\mathrm{H}$ & $\mathrm{H} \cdots A$ & $D \cdots A$ & $D-\mathrm{H} \cdots A$ \\
\hline $\mathrm{C} 10-\mathrm{H} 10 A \cdots \mathrm{O} 3$ & 0.97 & 2.52 & $3.184(2)$ & 125 \\
\hline $\mathrm{C} 12-\mathrm{H} 12 B \cdots \mathrm{O} 3$ & 0.97 & 2.46 & $3.135(2)$ & 126 \\
\hline $\mathrm{N} 1-\mathrm{H} 1 N \cdots \mathrm{O} 4^{\mathrm{i}}$ & 0.86 & 2.18 & $3.0217(19)$ & 168 \\
\hline $\mathrm{O} 4-\mathrm{H} 4 \mathrm{O} \cdots \mathrm{O}^{\mathrm{ii}}$ & 0.82 & 1.85 & $2.6619(18)$ & 174 \\
\hline $\mathrm{N} 6-\mathrm{H} 6 N \cdots \mathrm{O} 1$ & 0.86 & 2.00 & 2.8415 (19) & 166 \\
\hline $\mathrm{C} 10-\mathrm{H} 10 B \cdots \mathrm{O}^{\mathrm{iii}}$ & 0.97 & 2.49 & $3.413(2)$ & 159 \\
\hline $\mathrm{C} 15-\mathrm{H} 15 A \cdots \mathrm{O} 4^{\mathrm{iv}}$ & 0.97 & 2.50 & $3.160(2)$ & 125 \\
\hline $\mathrm{C} 20-\mathrm{H} 20 \cdots \mathrm{O} 5^{\mathrm{ii}}$ & 0.93 & 2.51 & $3.195(2)$ & 130 \\
\hline
\end{tabular}

Symmetry codes: (i) $x,-y+\frac{3}{2}, z+\frac{1}{2}$; (ii) $x,-y+\frac{1}{2}, z-\frac{1}{2}$; (iii) $-x+1, y-\frac{1}{2},-z+\frac{3}{2}$; (iv) $-x+1,-y+1,-z+1$.

CORCB-1 region due to the replacement of carboxylic acid with an ester functional group. As a result, a co-crystal former such as acetaminophen is needed for crystal formation to provide stable hydrogen bonds: with acetaminophen molecules flanking CORCB-1-APAP; no interactions are observed between these difunctionalized compounds. The cocrystal shows three classical hydrogen bonds. The first is an $\mathrm{N} 6-\mathrm{H} 6 N \cdots \mathrm{O} 1$ hydrogen bond $(\mathrm{H} \cdots \mathrm{O}=2.00 \AA)$ found between the $\mathrm{N}-\mathrm{H}$ group of acetaminophen and the $\mathrm{C}=\mathrm{O}$ acceptor from CORCB-1-APAP. This type of bond has been previously reported in the acetaminophen co-crystal with citric acid (Elbagerma et al., 2011). Pure acetaminophen crystals typically only form hydrogen bonds between $\mathrm{N}-$ $\mathrm{H} \cdots \mathrm{O}-\mathrm{H}$ and $\mathrm{O}-\mathrm{H} \cdots \mathrm{O}=\mathrm{C}$. The second interaction $\mathrm{N} 1-$ $\mathrm{H} 1 \mathrm{~N} \cdots \mathrm{O} 4-\mathrm{H} 4 \mathrm{O}$ is between CORCB-1-APAP and another

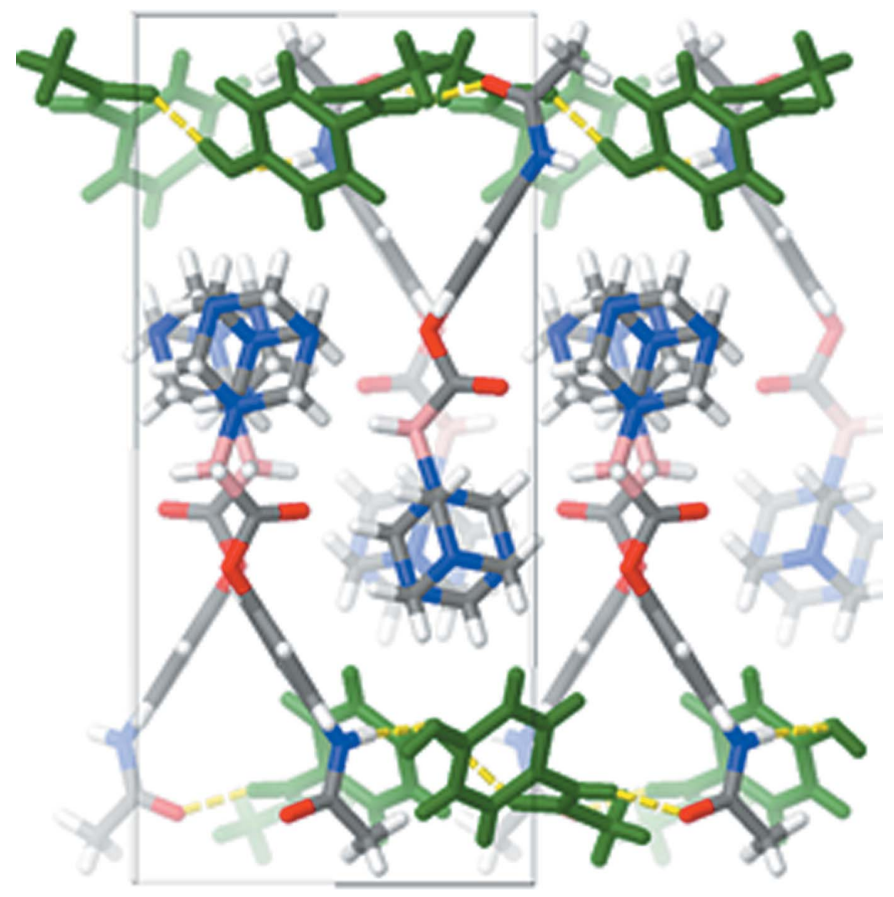

Figure 2

Unit cell packing of (I) viewed down the $c$-axis direction, with additional molecules added along the $b$-axis direction. Acetaminophen molecules are green; hydrogen bonds are shown as dashed yellow lines.

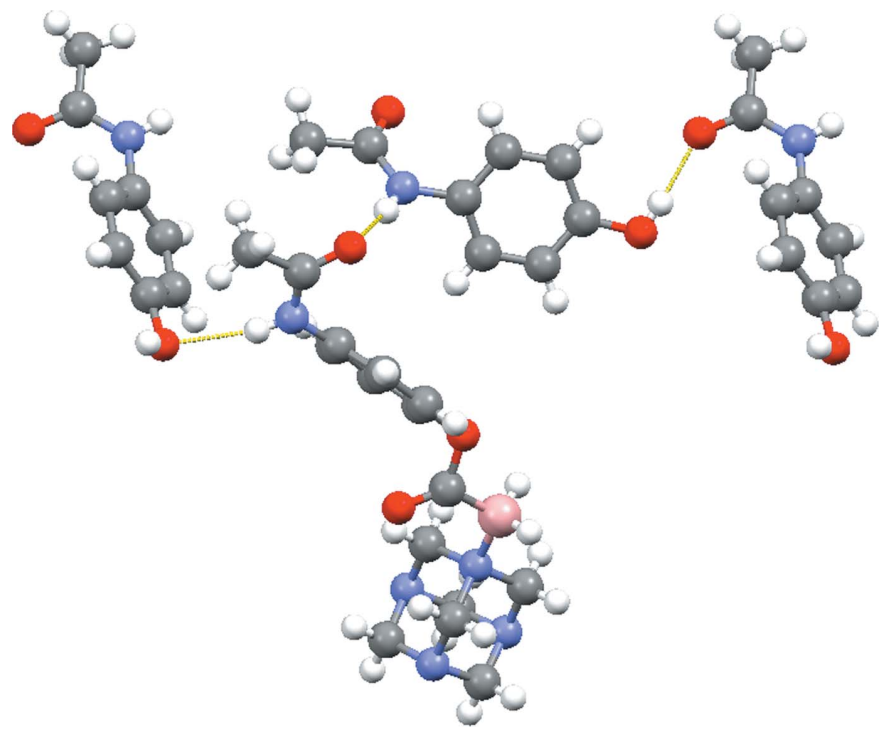

Figure 3

Detail of the packing of (I) showing hydrogen bonds (yellow lines) between the components of the co-crystal. Three acetaminophen molecules are shown but only the two on the left are hydrogen bonded with CORCB-1-APAP. The third acetaminophen molecule, which accepts a hydrogen bond from the second, is oriented in the same way as the first and repeats the pattern.

acetaminophen molecule. The bond length $(2.18 \AA)$ of this hydrogen bond is similar to the $\mathrm{N}-\mathrm{H} \cdots \mathrm{O}-\mathrm{H}$ bond $(2.09 \AA)$ from the known acetaminophen crystal form II (Agnew et al., 2016; Thomas et al., 2011). The third hydrogen bond does not involve CORCB-1-APAP: it is exclusively formed between two acetaminophen molecules and this $\mathrm{O} 4-\mathrm{H} 4 \mathrm{O} \cdots \mathrm{O} 5=\mathrm{C} 17$ bond $(1.85 \AA)$ is identical in length to that of acetaminophen crystal form II (1.85 $⿱$ ) ). Several weak $\mathrm{C}-\mathrm{H}$. . O hydrogen bonds may help to consolidate the structure.

A molecular packing projection of (I) is shown in Fig. 4 for clear representation of each pair of CORCB-1-APAP and its co-former, acetaminophen. As noted, the observed hydrogenbond lengths in this co-crystal are similar to those from acetaminophen form II packing while the overall packing looks similar to form I (Naumov et al., 1998).

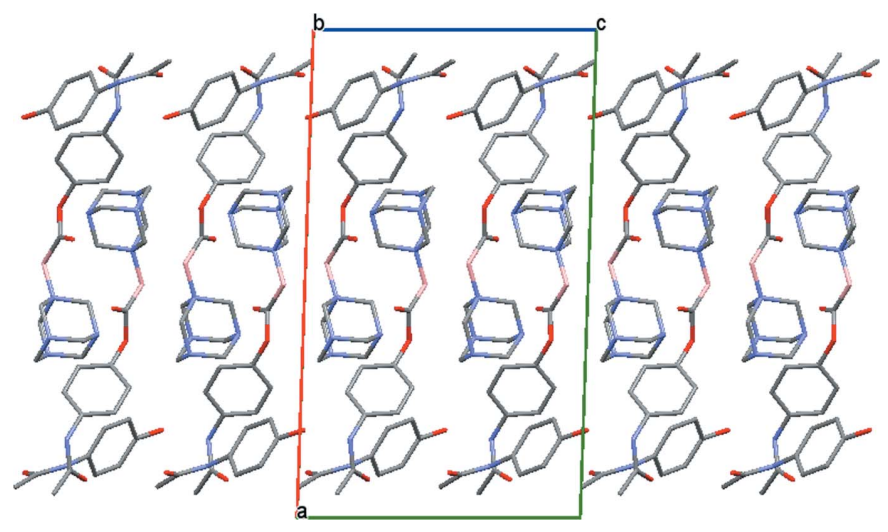

Figure 4

Molecular packing diagram for (I) viewed down [010]. 
Table 2

Experimental details.

\begin{tabular}{|c|c|}
\hline \multicolumn{2}{|l|}{ Crystal data } \\
\hline Chemical formula & $\mathrm{C}_{15} \mathrm{H}_{22} \mathrm{BN}_{5} \mathrm{O}_{3} \cdot \mathrm{C}_{8} \mathrm{H}_{9} \mathrm{NO}_{2}$ \\
\hline$M_{\mathrm{r}}$ & 482.35 \\
\hline Crystal system, space group & Monoclinic, $P 2_{1} / c$ \\
\hline Temperature $(\mathrm{K})$ & 293 \\
\hline$a, b, c(\AA)$ & $20.760(4), 9.5527(19), 12.045(2)$ \\
\hline$\beta\left({ }^{\circ}\right)$ & 91.929 (4) \\
\hline$V\left(\mathrm{~A}^{3}\right)$ & $2387.2(8)$ \\
\hline$Z$ & 4 \\
\hline Radiation type & Мо $K \alpha$ \\
\hline$\mu\left(\mathrm{mm}^{-1}\right)$ & 0.10 \\
\hline Crystal size $(\mathrm{mm})$ & $0.23 \times 0.06 \times 0.05$ \\
\hline \multicolumn{2}{|l|}{ Data collection } \\
\hline Diffractometer & Bruker APEXII CCD \\
\hline $\begin{array}{l}\text { No. of measured, independent and } \\
\text { observed }[I>2 \sigma(I)] \text { reflections }\end{array}$ & $29824,4870,3044$ \\
\hline$R_{\text {int }}$ & 0.091 \\
\hline$(\sin \theta / \lambda)_{\max }\left(\AA^{-1}\right)$ & 0.625 \\
\hline \multicolumn{2}{|l|}{ Refinement } \\
\hline$R\left[F^{2}>2 \sigma\left(F^{2}\right)\right], w R\left(F^{2}\right), S$ & $0.041,0.072,1.08$ \\
\hline No. of reflections & 4870 \\
\hline No. of parameters & 319 \\
\hline H-atom treatment & $\mathrm{H}$-atom parameters constrained \\
\hline$\Delta \rho_{\max }, \Delta \rho_{\min }\left(\mathrm{e} \AA^{-3}\right)$ & $0.24,-0.22$ \\
\hline
\end{tabular}

Computer programs: APEX2 and SAINT (Bruker, 2007), SHELXS97 (Sheldrick, 2008), SHELXL2014/7 (Sheldrick, 2015), SHELXTL (Sheldrick, 2008) and publCIF (Westrip, 2010).

\section{Database survey}

The crystal structures of amine carboxyborane have been reported as dimers (Spielvogel et al., 1980; Rana et al., 2002; Vyakaranam et al., 2002). The CORCB-1 crystal structure does not show typical hydrogen bonding from carboxylic acid groups and does not show dimer formation (Ayudhya et al., 2017). Acetaminophen co-crystallized structures to name a few are with ibuprofen (Stone et al., 2009), citric acid (Elbagerma et al., 2011), theophylline (Childs et al., 2007) and morpholine (Oswald et al., 2002).

\section{Synthesis and crystallization}

The synthesis of amine carboxyborane derivatives such as methyl ester of various amine carboxyborates have been described previously. Several esterification methods of amine carboxyboranes with alcohols include using DCC to make 98\% yield (Spielvogel et al., 1986) and using a catalytic amount of hydrogen bromide, which provides nearly quantitative yields (Györi et al., 1995). In our process, esterification is completed before the amine exchange reaction and not vice versa. The synthesis of hexamethylenetetramine carboacetaminophenborane (CORCB-1-APAP) involves several steps using trimethylamine carboxyborane (CORCB-3) as the starting material. Trimethylamine carboxyborane, synthesized by the previously reported method (Spielvogel et al., 1976) is first esterified at the carboxyborate moiety with acetaminophen (APAP). The esterification was carried out in a mixed solvent system of chloroform and THF (1:1) at 313 to $318 \mathrm{~K}$ for five days and the crude product was purified by a series of recrystallizations. CORCB-1-APAP and acetaminophen cocrystals for X-ray data collection were grown in mixed solvents of hexane/chloroform using the solution crystallization method.

\section{Refinement}

Crystal data, data collection and structure refinement details are summarized in Table 2. All hydrogen atoms were treated as riding atoms in geometrically idealized positions $[\mathrm{N}-\mathrm{H}=$ $0.86, \mathrm{O}-\mathrm{H}=0.82$ and $\mathrm{C}-\mathrm{H}=0.93-0.97 \AA$ with $U_{\text {iso }}(\mathrm{H})=$ $1.2 U_{\mathrm{eq}}(\mathrm{N}, \mathrm{O}, \mathrm{C})$ or $1.2 U_{\mathrm{eq}}($ Cmethyl $\left.)\right]$.

\section{Acknowledgements}

The authors thank M. Zeller for the X-ray data collection and the NSF for funding the diffractometer (DMR-1337296) at Youngstown State University.

\section{References}

Agnew, L. R., Cruickshank, D. L., McGlone, T. \& Wilson, C. C. (2016). Chem. Commun. 52, 7368-7371.

Ayudhya, T. I., Pellechia, P. J. \& Dingra, N. N. (2018). Dalton Trans. 47, 538-543.

Ayudhya, T. I., Raymond, C. C. \& Dingra, N. N. (2017). Dalton Trans. 46, 882-889.

Bruker (2007). APEX2 and SAINT. Bruker AXS Inc., Madison, Wisconsin, USA.

Cheney, M. L., Weyna, D. R., Shan, N., Hanna, M., Wojtas, L. \& Zaworotko, M. J. (2011). J. Pharm. Sci. 100, 2172-2181.

Childs, S. L., Stahly, G. P. \& Park, A. (2007). Mol. Pharm. 4, 323-338.

Dalpiaz, A., Ferretti, V., Bertolasi, V., Pavan, B., Monari, A. \& Pastore, M. (2018). Mol. Pharm. 15, 268-278.

Das, M. K., Mukherjee, P. \& Roy, S. (1990). Bull. Chem. Soc. Jpn, 63, 3658-3660.

Desiraju, G. R. (2007). Angew. Chem. Int. Ed. 46, 8342-8356.

Elbagerma, M. A., Edwards, H. G. M., Munshi, T. \& Scowen, I. J. (2011). CrystEngComm, 13, 1877-1884.

Groom, C. R., Bruno, I. J., Lightfoot, M. P. \& Ward, S. C. (2016). Acta Cryst. B72, 171-179.

Győri, B., Berente, Z., Emri, J. \& Lázár, I. (1995). Synthesis, 1995, 191-194.

Hall, I. H., Rajendran, K. G., Chen, S. Y., Wong, O. T., Sood, A. \& Spielvogel, B. F. (1995). Arch. Pharm. Pharm. Med. Chem. 328, 3944.

Hall, I. H., Spielvogel, B. F. \& Sood, A. (1990). Anticancer Drugs, 1, 133-142.

Kaur, R., Cavanagh, K. L., Rodríguez-Hornedo, N. \& Matzger, A. J. (2017). Cryst. Growth Des. 17, 5012-5016.

Murphy, M. E., Elkins, A. L., Shrewsbury, R. P., Sood, A., Spielvogel, B. F. \& Hall, I. H. (1996). Met.-Based Drugs, 3, 31-47.

Naumov, D. Yu., Vasilchenko, M. A. \& Howard, J. A. K. (1998). Acta Cryst. C54, 653-655.

Nugrahani, I., Asyarie, S., Soewandhi, S. N. \& Ibrahim, S. (2007). Int. J. Pharm. 3, 475-481.

Oswald, I. D. H., Allan, D. R., McGregor, P. A., Motherwell, W. D. S., Parsons, S. \& Pulham, C. R. (2002). Acta Cryst. B58, 1057-1066.

Rana, G., Vyakaranam, K., Zheng, C., Li, S., Spielvogel, B. F. \& Hosmane, N. S. (2002). Main Group Met. Chem. 25, 179-180.

Sheldrick, G. M. (2008). Acta Cryst. A64, 112-122.

Sheldrick, G. M. (2015). Acta Cryst. C71, 3-8.

Spielvogel, B. F., Ahmed, F. U. \& Mcphail, A. T. (1986). Synthesis, 1986, 833-835. 
Spielvogel, B. F., Das, M. K., McPhail, A. T., Onan, K. D. \& Hall, I. H. (1980). J. Am. Chem. Soc. 102, 6343-6344.

Spielvogel, B. F., Wojnowich, L., Das, M. K., McPhail, A. T. \& Hargrave, K. D. (1976). J. Am. Chem. Soc. 98, 5702-5703.

Stone, K. H., Lapidus, S. H. \& Stephens, P. W. (2009). J. Appl. Cryst. 42, 385-391.
Thomas, L. H., Wales, C., Zhao, L. \& Wilson, C. C. (2011). Cryst. Growth Des. 11, 1450-1452.

Vyakaranam, K., Rana, G., Chong, G., Zheng, S. L., Spielvogel, B. F. \& Hosmane, N. S. (2002). Main Group Met. Chem. 25, 181-182. Westrip, S. P. (2010). J. Appl. Cryst. 43, 920-925. 


\section{supporting information}

Acta Cryst. (2020). E76, 1854-1858 [https://doi.org/10.1107/S2056989020015327]

Synthesis and structure of a 1:1 co-crystal of hexamethylenetetramine carboxyborane and acetaminophen

\section{Theppawut Ayudhya, Casey Raymond and Nin Dingra}

Computing details

Data collection: APEX2 (Bruker, 2007); cell refinement: SAINT (Bruker, 2007); data reduction: SAINT (Bruker, 2007); program(s) used to solve structure: SHELXS97 (Sheldrick, 2008); program(s) used to refine structure: SHELXL2014/7 (Sheldrick, 2015); molecular graphics: SHELXTL (Sheldrick, 2008); software used to prepare material for publication: SHELXTL (Sheldrick, 2008) and publCIF (Westrip, 2010).

Hexamethylenetetramine 4-acetamidophenyl 2-boranylacetate-4-acetamidophenol (1/1)

Crystal data

$\mathrm{C}_{15} \mathrm{H}_{22} \mathrm{BN}_{5} \mathrm{O}_{3} \cdot \mathrm{C}_{8} \mathrm{H}_{9} \mathrm{NO}_{2}$

$M_{r}=482.35$

Monoclinic, $P 2_{1} / c$

$a=20.760(4) \AA$

$b=9.5527(19) \AA$

$c=12.045(2) \AA$

$\beta=91.929(4)^{\circ}$

$V=2387.2(8) \AA^{3}$

$Z=4$

\section{Data collection}

Bruker APEXII CCD diffractometer

Radiation source: sealed tube phi and $\omega$ scans 29824 measured reflections 4870 independent reflections

\section{Refinement}

Refinement on $F^{2}$

Least-squares matrix: full

$R\left[F^{2}>2 \sigma\left(F^{2}\right)\right]=0.041$

$w R\left(F^{2}\right)=0.072$

$S=1.08$

4870 reflections

319 parameters

0 restraints
$F(000)=1024$

$D_{\mathrm{x}}=1.342 \mathrm{Mg} \mathrm{m}^{-3}$

Mo $K \alpha$ radiation, $\lambda=0.71073 \AA$

Cell parameters from 7373 reflections

$\theta=5.8-54.1^{\circ}$

$\mu=0.10 \mathrm{~mm}^{-1}$

$T=293 \mathrm{~K}$

Needle, colorless

$0.23 \times 0.06 \times 0.05 \mathrm{~mm}$

3044 reflections with $I>2 \sigma(I)$

$R_{\text {int }}=0.091$

$\theta_{\text {max }}=26.4^{\circ}, \theta_{\text {min }}=2.0^{\circ}$

$h=-25 \rightarrow 25$

$k=-11 \rightarrow 11$

$l=-15 \rightarrow 15$

Primary atom site location: structure-invariant direct methods

Hydrogen site location: inferred from neighbouring sites

H-atom parameters constrained

$w=1 /\left[\sigma^{2}\left(F_{\mathrm{o}}^{2}\right)+(0.018 P)^{2}\right]$

where $P=\left(F_{\mathrm{o}}{ }^{2}+2 F_{\mathrm{c}}{ }^{2}\right) / 3$

$(\Delta / \sigma)_{\max }<0.001$

$\Delta \rho_{\max }=0.24 \mathrm{e}^{-3}$

$\Delta \rho_{\min }=-0.22$ e $\AA^{-3}$ 


\section{Special details}

Geometry. All esds (except the esd in the dihedral angle between two 1.s. planes) are estimated using the full covariance matrix. The cell esds are taken into account individually in the estimation of esds in distances, angles and torsion angles; correlations between esds in cell parameters are only used when they are defined by crystal symmetry. An approximate (isotropic) treatment of cell esds is used for estimating esds involving l.s. planes.

Fractional atomic coordinates and isotropic or equivalent isotropic displacement parameters $\left(\AA^{2}\right)$

\begin{tabular}{|c|c|c|c|c|}
\hline & $x$ & $y$ & $z$ & $U_{\text {iso }} * / U_{\text {eq }}$ \\
\hline $\mathrm{C} 1$ & $0.05099(9)$ & $1.08726(18)$ & $0.86077(16)$ & $0.0285(5)$ \\
\hline $\mathrm{H} 1 \mathrm{~A}$ & 0.0175 & 1.1125 & 0.8076 & $0.043 *$ \\
\hline H1B & 0.0732 & 1.1701 & 0.8859 & $0.043^{*}$ \\
\hline $\mathrm{H} 1 \mathrm{C}$ & 0.0324 & 1.0413 & 0.9229 & $0.043^{*}$ \\
\hline $\mathrm{C} 2$ & $0.09783(9)$ & 0.98985 (19) & $0.80733(15)$ & $0.0227(4)$ \\
\hline $\mathrm{O} 1$ & $0.07872(6)$ & $0.88651(12)$ & $0.75419(10)$ & $0.0260(3)$ \\
\hline N1 & $0.16095(7)$ & $1.02002(14)$ & $0.82219(12)$ & $0.0228(4)$ \\
\hline $\mathrm{H} 1 \mathrm{~N}$ & 0.1715 & 1.0898 & 0.8640 & $0.027 *$ \\
\hline $\mathrm{C} 3$ & $0.21163(8)$ & $0.94201(17)$ & $0.77195(15)$ & $0.0194(4)$ \\
\hline $\mathrm{C} 4$ & $0.25773(9)$ & $0.87339(17)$ & $0.83793(16)$ & $0.0221(4)$ \\
\hline $\mathrm{H} 4$ & 0.2543 & 0.8727 & 0.9147 & $0.026^{*}$ \\
\hline $\mathrm{C} 5$ & $0.30902(9)$ & $0.80567(17)$ & $0.78963(15)$ & $0.0217(5)$ \\
\hline H5 & 0.3401 & 0.7601 & 0.8337 & $0.026^{*}$ \\
\hline C6 & $0.31331(8)$ & 0.80687 (17) & $0.67507(16)$ & $0.0186(4)$ \\
\hline $\mathrm{C} 7$ & $0.26699(8)$ & $0.87268(17)$ & $0.60850(15)$ & 0.0209 (4) \\
\hline H7 & 0.2700 & 0.8718 & 0.5316 & $0.025^{*}$ \\
\hline $\mathrm{C} 8$ & $0.21585(9)$ & $0.94021(17)$ & $0.65765(15)$ & $0.0214(4)$ \\
\hline $\mathrm{H} 8$ & 0.1844 & 0.9843 & 0.6134 & $0.026^{*}$ \\
\hline $\mathrm{O} 2$ & $0.36389(6)$ & $0.73601(11)$ & $0.62473(10)$ & $0.0221(3)$ \\
\hline C9 & $0.42431(8)$ & 0.80105 (18) & $0.63013(15)$ & $0.0189(4)$ \\
\hline $\mathrm{O} 3$ & $0.42901(6)$ & $0.91608(12)$ & $0.67315(10)$ & $0.0242(3)$ \\
\hline B1 & $0.47824(10)$ & $0.7044(2)$ & $0.57512(19)$ & $0.0210(5)$ \\
\hline H1B1 & 0.4721 & 0.6078 & 0.5971 & $0.025^{*}$ \\
\hline H1B2 & 0.4735 & 0.7095 & 0.4948 & $0.025^{*}$ \\
\hline N2 & $0.54897(7)$ & $0.75441(14)$ & 0.61337 (11) & $0.0164(3)$ \\
\hline $\mathrm{C} 10$ & $0.55901(8)$ & $0.75507(18)$ & 0.73918 (14) & $0.0191(4)$ \\
\hline $\mathrm{H} 10 \mathrm{~A}$ & 0.5288 & 0.8198 & 0.7712 & $0.023 *$ \\
\hline H10B & 0.5502 & 0.6624 & 0.7679 & $0.023^{*}$ \\
\hline N3 & $0.62411(7)$ & $0.79543(14)$ & $0.77251(12)$ & $0.0206(4)$ \\
\hline C11 & $0.63675(9)$ & $0.93610(17)$ & $0.72684(15)$ & $0.0236(5)$ \\
\hline H11A & 0.6065 & 1.0022 & 0.7571 & $0.028^{*}$ \\
\hline H11B & 0.6799 & 0.9653 & 0.7502 & $0.028 *$ \\
\hline N4 & $0.63096(7)$ & 0.93989 (14) & $0.60505(12)$ & $0.0197(4)$ \\
\hline C12 & $0.56578(8)$ & 0.89959 (17) & $0.57118(15)$ & $0.0191(4)$ \\
\hline $\mathrm{H} 12 \mathrm{~A}$ & 0.5615 & 0.9008 & 0.4907 & $0.023^{*}$ \\
\hline H12B & 0.5356 & 0.9672 & 0.5997 & $0.023^{*}$ \\
\hline $\mathrm{C} 13$ & $0.59876(8)$ & $0.65314(17)$ & $0.56842(15)$ & $0.0209(4)$ \\
\hline H13A & 0.5903 & 0.5597 & 0.5958 & $0.025^{*}$ \\
\hline H13B & 0.5947 & 0.6509 & 0.4880 & $0.025^{*}$ \\
\hline
\end{tabular}




$\begin{array}{lllll}\text { N5 } & 0.66398(7) & 0.69366(14) & 0.60153(12) & 0.0202(4) \\ \text { C14 } & 0.66971(9) & 0.69583(18) & 0.72354(15) & 0.0234(5) \\ \text { H14A } & 0.7134 & 0.7217 & 0.7462 & 0.028^{*} \\ \text { H14B } & 0.6615 & 0.6026 & 0.7518 & 0.028^{*} \\ \text { C15 } & 0.67633(9) & 0.83683(18) & 0.56036(16) & 0.0244(5) \\ \text { H15A } & 0.7201 & 0.8639 & 0.5816 & 0.029^{*} \\ \text { H15B } & 0.6725 & 0.8372 & 0.4799 & 0.029^{*} \\ \text { C16 } & 0.06429(10) & 0.69737(19) & 0.99581(16) & 0.0331(5) \\ \text { H16A } & 0.0271 & 0.6676 & 1.0348 & 0.050^{*} \\ \text { H16B } & 0.0528 & 0.7742 & 0.9477 & 0.050^{*} \\ \text { H16C } & 0.0974 & 0.7267 & 1.0484 & 0.050^{*} \\ \text { C17 } & 0.08889(9) & 0.57733(19) & 0.92747(16) & 0.0244(5) \\ \text { O5 } & 0.09247(6) & 0.45723(12) & 0.96712(10) & 0.0300(3) \\ \text { N6 } & 0.10633(7) & 0.60827(14) & 0.82433(12) & 0.0243(4) \\ \text { H6N } & 0.1048 & 0.6947 & 0.8043 & 0.029^{*} \\ \text { C18 } & 0.12735(9) & 0.50769(17) & 0.74469(15) & 0.0211(4) \\ \text { C19 } & 0.08895(9) & 0.39203(18) & 0.71821(15) & 0.0227(4) \\ \text { H19 } & 0.0513 & 0.3762 & 0.7559 & 0.027^{*} \\ \text { C20 } & 0.10712(9) & 0.30031(18) & 0.63528(15) & 0.0232(5) \\ \text { H20 } & 0.0820 & 0.2221 & 0.6183 & 0.028^{*} \\ \text { C21 } & 0.16282(9) & 0.32553(18) & 0.57793(15) & 0.0213(4) \\ \text { O4 } & 0.17995(6) & 0.24556(12) & 0.48852(11) & 0.0272(3) \\ \text { H4O } & 0.1529 & 0.1843 & 0.4771 & 0.033^{*} \\ \text { C22 } & 0.20183(9) & 0.43896(18) & 0.60524(15) & 0.0242(5) \\ \text { H22 } & 0.2398 & 0.4542 & 0.5681 & 0.029^{*} \\ \text { C23 } & 0.18357(9) & 0.53024(18) & 0.68920(15) & 0.0238(5) \\ \text { H23 } & 0.2095 & 0.6067 & 0.7078 & 0.029^{*}\end{array}$

Atomic displacement parameters $\left(\AA^{2}\right)$

\begin{tabular}{lllllll}
\hline & $U^{11}$ & $U^{22}$ & $U^{33}$ & $U^{12}$ & $U^{13}$ & $U^{23}$ \\
\hline C1 & $0.0260(12)$ & $0.0238(10)$ & $0.0363(13)$ & $0.0017(9)$ & $0.0109(10)$ & $-0.0002(9)$ \\
C2 & $0.0258(12)$ & $0.0189(10)$ & $0.0237(12)$ & $0.0016(9)$ & $0.0041(9)$ & $0.0036(9)$ \\
O1 & $0.0257(8)$ & $0.0202(7)$ & $0.0320(8)$ & $-0.0011(6)$ & $0.0027(6)$ & $-0.0018(6)$ \\
N1 & $0.0211(9)$ & $0.0188(8)$ & $0.0286(10)$ & $0.0010(7)$ & $0.0035(8)$ & $-0.0056(7)$ \\
C3 & $0.0181(11)$ & $0.0149(10)$ & $0.0254(12)$ & $-0.0012(8)$ & $0.0047(9)$ & $-0.0010(8)$ \\
C4 & $0.0274(12)$ & $0.0188(10)$ & $0.0203(11)$ & $-0.0021(9)$ & $0.0053(9)$ & $0.0014(8)$ \\
C5 & $0.0230(11)$ & $0.0159(10)$ & $0.0262(12)$ & $0.0018(8)$ & $0.0005(9)$ & $0.0032(8)$ \\
C6 & $0.0155(10)$ & $0.0115(9)$ & $0.0292(12)$ & $-0.0014(8)$ & $0.0069(9)$ & $-0.0016(8)$ \\
C7 & $0.0259(11)$ & $0.0175(10)$ & $0.0195(11)$ & $-0.0039(8)$ & $0.0032(9)$ & $-0.0016(8)$ \\
C8 & $0.0201(11)$ & $0.0178(10)$ & $0.0260(12)$ & $0.0014(8)$ & $-0.0029(9)$ & $0.0015(8)$ \\
O2 & $0.0177(7)$ & $0.0171(7)$ & $0.0316(8)$ & $-0.0019(5)$ & $0.0048(6)$ & $-0.0060(6)$ \\
C9 & $0.0174(10)$ & $0.0182(10)$ & $0.0213(11)$ & $-0.0004(8)$ & $0.0017(9)$ & $0.0033(8)$ \\
O3 & $0.0208(7)$ & $0.0172(7)$ & $0.0349(8)$ & $-0.0014(6)$ & $0.0043(6)$ & $-0.0062(6)$ \\
B1 & $0.0214(13)$ & $0.0172(11)$ & $0.0246(13)$ & $-0.0034(9)$ & $0.0028(10)$ & $-0.0016(9)$ \\
N2 & $0.0197(9)$ & $0.0131(7)$ & $0.0168(9)$ & $0.0019(6)$ & $0.0044(7)$ & $-0.0007(6)$ \\
C10 & $0.0231(11)$ & $0.0170(9)$ & $0.0174(11)$ & $0.0035(8)$ & $0.0043(9)$ & $0.0020(8)$ \\
N3 & $0.0205(9)$ & $0.0206(8)$ & $0.0209(9)$ & $0.0048(7)$ & $0.0004(7)$ & $0.0010(7)$
\end{tabular}




\begin{tabular}{lllllll} 
& & & & & \\
C11 & $0.0201(11)$ & $0.0193(10)$ & $0.0312(12)$ & $0.0006(8)$ & $-0.0001(9)$ & $-0.0042(9)$ \\
N4 & $0.0167(9)$ & $0.0174(8)$ & $0.0252(10)$ & $0.0010(7)$ & $0.0034(7)$ & $0.0032(7)$ \\
C12 & $0.0212(11)$ & $0.0157(9)$ & $0.0206(11)$ & $0.0002(8)$ & $0.0025(9)$ & $0.0053(8)$ \\
C13 & $0.0221(11)$ & $0.0180(10)$ & $0.0231(11)$ & $0.0035(8)$ & $0.0071(9)$ & $-0.0027(8)$ \\
N5 & $0.0171(9)$ & $0.0197(8)$ & $0.0239(10)$ & $0.0017(7)$ & $0.0047(7)$ & $0.0013(7)$ \\
C14 & $0.0212(11)$ & $0.0215(10)$ & $0.0274(12)$ & $0.0041(8)$ & $0.0001(9)$ & $0.0030(9)$ \\
C15 & $0.0192(11)$ & $0.0244(11)$ & $0.0298(12)$ & $0.0004(8)$ & $0.0061(9)$ & $0.0042(9)$ \\
C16 & $0.0420(14)$ & $0.0275(11)$ & $0.0299(13)$ & $0.0095(10)$ & $0.0036(11)$ & $0.0009(9)$ \\
C17 & $0.0259(12)$ & $0.0228(11)$ & $0.0245(12)$ & $0.0017(9)$ & $-0.0014(10)$ & $0.0003(9)$ \\
O5 & $0.0389(9)$ & $0.0225(7)$ & $0.0287(8)$ & $0.0038(6)$ & $0.0033(7)$ & $0.0040(6)$ \\
N6 & $0.0338(10)$ & $0.0145(8)$ & $0.0247(10)$ & $0.0014(7)$ & $0.0018(8)$ & $0.0004(7)$ \\
C18 & $0.0241(11)$ & $0.0176(10)$ & $0.0216(11)$ & $0.0022(9)$ & $-0.0009(9)$ & $0.0010(8)$ \\
C19 & $0.0205(11)$ & $0.0209(10)$ & $0.0270(12)$ & $0.0009(8)$ & $0.0037(9)$ & $0.0024(9)$ \\
C20 & $0.0230(11)$ & $0.0171(10)$ & $0.0296(12)$ & $-0.0026(8)$ & $0.0035(10)$ & $0.0000(9)$ \\
C21 & $0.0239(11)$ & $0.0166(10)$ & $0.0236(12)$ & $0.0047(8)$ & $0.0013(9)$ & $0.0018(8)$ \\
O4 & $0.0275(8)$ & $0.0218(7)$ & $0.0326(8)$ & $-0.0007(6)$ & $0.0080(7)$ & $-0.0037(6)$ \\
C22 & $0.0203(11)$ & $0.0249(11)$ & $0.0275(12)$ & $-0.0018(9)$ & $0.0013(9)$ & $0.0047(9)$ \\
C23 & $0.0241(12)$ & $0.0199(10)$ & $0.0271(12)$ & $-0.0044(8)$ & $-0.0028(10)$ & $0.0020(9)$ \\
& & & & & & \\
\hline
\end{tabular}

Geometric parameters $\left(\AA,{ }^{o}\right)$

\begin{tabular}{llll}
\hline $\mathrm{C} 1-\mathrm{C} 2$ & $1.506(2)$ & $\mathrm{C} 11-\mathrm{H} 11 \mathrm{~B}$ & 0.9700 \\
$\mathrm{C} 1-\mathrm{H} 1 \mathrm{~A}$ & 0.9600 & $\mathrm{~N} 4-\mathrm{C} 12$ & $1.452(2)$ \\
$\mathrm{C} 1-\mathrm{H} 1 \mathrm{~B}$ & 0.9600 & $\mathrm{~N} 4-\mathrm{C} 15$ & $1.477(2)$ \\
$\mathrm{C} 1-\mathrm{H} 1 \mathrm{C}$ & 0.9600 & $\mathrm{C} 12-\mathrm{H} 12 \mathrm{~A}$ & 0.9700 \\
$\mathrm{C} 2-\mathrm{O} 1$ & $1.235(2)$ & $\mathrm{C} 12-\mathrm{H} 12 \mathrm{~B}$ & 0.9700 \\
$\mathrm{C} 2-\mathrm{N} 1$ & $1.348(2)$ & $\mathrm{C} 13-\mathrm{N} 5$ & $1.451(2)$ \\
$\mathrm{N} 1-\mathrm{C} 3$ & $1.439(2)$ & $\mathrm{C} 13-\mathrm{H} 13 \mathrm{~A}$ & 0.9700 \\
$\mathrm{~N} 1-\mathrm{H} 1 \mathrm{~N}$ & 0.8600 & $\mathrm{C} 13-\mathrm{H} 13 \mathrm{~B}$ & 0.9700 \\
$\mathrm{C} 3-\mathrm{C} 8$ & $1.383(2)$ & $\mathrm{N} 5-\mathrm{C} 14$ & $1.471(2)$ \\
$\mathrm{C} 3-\mathrm{C} 4$ & $1.388(2)$ & $\mathrm{N} 5-\mathrm{C} 15$ & $1.480(2)$ \\
$\mathrm{C} 4-\mathrm{C} 5$ & $1.390(2)$ & $\mathrm{C} 14-\mathrm{H} 14 \mathrm{~A}$ & 0.9700 \\
$\mathrm{C} 4-\mathrm{H} 4$ & 0.9300 & $\mathrm{C} 14-\mathrm{H} 14 \mathrm{~B}$ & 0.9700 \\
$\mathrm{C} 5-\mathrm{C} 6$ & $1.386(2)$ & $\mathrm{C} 15-\mathrm{H} 15 \mathrm{~A}$ & 0.9700 \\
$\mathrm{C} 5-\mathrm{H} 5$ & 0.9300 & $\mathrm{C} 15-\mathrm{H} 15 \mathrm{~B}$ & 0.9700 \\
$\mathrm{C} 6-\mathrm{C} 7$ & $1.382(2)$ & $\mathrm{C} 16-\mathrm{C} 17$ & $1.511(2)$ \\
$\mathrm{C} 6-\mathrm{O} 2$ & $1.404(2)$ & $\mathrm{C} 16-\mathrm{H} 16 \mathrm{~A}$ & 0.9600 \\
$\mathrm{C} 7-\mathrm{C} 8$ & $1.391(2)$ & $\mathrm{C} 16-\mathrm{H} 16 \mathrm{~B}$ & 0.9600 \\
$\mathrm{C} 7-\mathrm{H} 7$ & 0.9300 & $\mathrm{C} 16-\mathrm{H} 16 \mathrm{C}$ & 0.9600 \\
$\mathrm{C} 8-\mathrm{H} 8$ & 0.9300 & $\mathrm{C} 17-\mathrm{O} 5$ & $1.244(2)$ \\
$\mathrm{O} 2-\mathrm{C} 9$ & $1.399(2)$ & $\mathrm{C} 17-\mathrm{N} 6$ & $1.339(2)$ \\
$\mathrm{C} 9-\mathrm{O} 3$ & $1.2174(19)$ & $\mathrm{N} 6-\mathrm{C} 18$ & $1.436(2)$ \\
$\mathrm{C} 9-\mathrm{B} 1$ & $1.611(3)$ & $\mathrm{N} 6-\mathrm{H} 6 \mathrm{~N}$ & 0.8600 \\
$\mathrm{~B} 1-\mathrm{N} 2$ & $1.597(2)$ & $\mathrm{C} 18-\mathrm{C} 23$ & $1.381(2)$ \\
$\mathrm{B} 1-\mathrm{H} 1 \mathrm{~B} 1$ & 0.9700 & $\mathrm{C} 18-\mathrm{C} 19$ & $1.393(2)$ \\
$\mathrm{B} 1-\mathrm{H} 1 \mathrm{~B} 2$ & 0.9700 & $\mathrm{C} 19-\mathrm{C} 20$ & $1.390(2)$ \\
$\mathrm{N} 2-\mathrm{C} 10$ & $1.523(2)$ & $\mathrm{C} 19-\mathrm{H} 19-\mathrm{C} 21$ & 0.9300 \\
$\mathrm{~N} 2-\mathrm{C} 12$ & $1.522(2)$ & & $1.388(2)$ \\
& &
\end{tabular}




\begin{tabular}{|c|c|c|c|}
\hline $\mathrm{N} 2-\mathrm{C} 13$ & $1.528(2)$ & $\mathrm{C} 20-\mathrm{H} 20$ & 0.9300 \\
\hline $\mathrm{C} 10-\mathrm{N} 3$ & $1.449(2)$ & $\mathrm{C} 21-\mathrm{O} 4$ & $1.377(2)$ \\
\hline $\mathrm{C} 10-\mathrm{H} 10 \mathrm{~A}$ & 0.9700 & $\mathrm{C} 21-\mathrm{C} 22$ & $1.386(2)$ \\
\hline $\mathrm{C} 10-\mathrm{H} 10 \mathrm{~B}$ & 0.9700 & $\mathrm{O} 4-\mathrm{H} 4 \mathrm{O}$ & 0.8200 \\
\hline N3-C11 & $1.479(2)$ & $\mathrm{C} 22-\mathrm{C} 23$ & $1.397(2)$ \\
\hline N3-C14 & $1.479(2)$ & $\mathrm{C} 22-\mathrm{H} 22$ & 0.9300 \\
\hline $\mathrm{C} 11-\mathrm{N} 4$ & $1.468(2)$ & $\mathrm{C} 23-\mathrm{H} 23$ & 0.9300 \\
\hline $\mathrm{C} 11-\mathrm{H} 11 \mathrm{~A}$ & 0.9700 & & \\
\hline $\mathrm{C} 2-\mathrm{C} 1-\mathrm{H} 1 \mathrm{~A}$ & 109.5 & $\mathrm{C} 12-\mathrm{N} 4-\mathrm{C} 11$ & $108.57(14)$ \\
\hline $\mathrm{C} 2-\mathrm{C} 1-\mathrm{H} 1 \mathrm{~B}$ & 109.5 & $\mathrm{C} 12-\mathrm{N} 4-\mathrm{C} 15$ & $108.70(14)$ \\
\hline $\mathrm{H} 1 \mathrm{~A}-\mathrm{C} 1-\mathrm{H} 1 \mathrm{~B}$ & 109.5 & $\mathrm{C} 11-\mathrm{N} 4-\mathrm{C} 15$ & $108.42(14)$ \\
\hline $\mathrm{C} 2-\mathrm{C} 1-\mathrm{H} 1 \mathrm{C}$ & 109.5 & $\mathrm{~N} 4-\mathrm{C} 12-\mathrm{N} 2$ & $111.71(13)$ \\
\hline $\mathrm{H} 1 \mathrm{~A}-\mathrm{C} 1-\mathrm{H} 1 \mathrm{C}$ & 109.5 & $\mathrm{~N} 4-\mathrm{C} 12-\mathrm{H} 12 \mathrm{~A}$ & 109.3 \\
\hline $\mathrm{H} 1 \mathrm{~B}-\mathrm{C} 1-\mathrm{H} 1 \mathrm{C}$ & 109.5 & $\mathrm{~N} 2-\mathrm{C} 12-\mathrm{H} 12 \mathrm{~A}$ & 109.3 \\
\hline $\mathrm{O} 1-\mathrm{C} 2-\mathrm{N} 1$ & $122.26(17)$ & $\mathrm{N} 4-\mathrm{C} 12-\mathrm{H} 12 \mathrm{~B}$ & 109.3 \\
\hline $\mathrm{O} 1-\mathrm{C} 2-\mathrm{C} 1$ & $120.98(17)$ & $\mathrm{N} 2-\mathrm{C} 12-\mathrm{H} 12 \mathrm{~B}$ & 109.3 \\
\hline $\mathrm{N} 1-\mathrm{C} 2-\mathrm{C} 1$ & $116.74(16)$ & $\mathrm{H} 12 \mathrm{~A}-\mathrm{C} 12-\mathrm{H} 12 \mathrm{~B}$ & 107.9 \\
\hline $\mathrm{C} 2-\mathrm{N} 1-\mathrm{C} 3$ & $123.69(15)$ & $\mathrm{N} 5-\mathrm{C} 13-\mathrm{N} 2$ & $111.70(13)$ \\
\hline $\mathrm{C} 2-\mathrm{N} 1-\mathrm{H} 1 \mathrm{~N}$ & 118.2 & $\mathrm{~N} 5-\mathrm{C} 13-\mathrm{H} 13 \mathrm{~A}$ & 109.3 \\
\hline $\mathrm{C} 3-\mathrm{N} 1-\mathrm{H} 1 \mathrm{~N}$ & 118.2 & $\mathrm{~N} 2-\mathrm{C} 13-\mathrm{H} 13 \mathrm{~A}$ & 109.3 \\
\hline $\mathrm{C} 8-\mathrm{C} 3-\mathrm{C} 4$ & $119.91(17)$ & N5-C13-H13B & 109.3 \\
\hline $\mathrm{C} 8-\mathrm{C} 3-\mathrm{N} 1$ & $119.80(16)$ & $\mathrm{N} 2-\mathrm{C} 13-\mathrm{H} 13 \mathrm{~B}$ & 109.3 \\
\hline $\mathrm{C} 4-\mathrm{C} 3-\mathrm{N} 1$ & $120.24(16)$ & $\mathrm{H} 13 \mathrm{~A}-\mathrm{C} 13-\mathrm{H} 13 \mathrm{~B}$ & 107.9 \\
\hline $\mathrm{C} 5-\mathrm{C} 4-\mathrm{C} 3$ & $120.23(17)$ & $\mathrm{C} 13-\mathrm{N} 5-\mathrm{C} 14$ & $108.77(14)$ \\
\hline $\mathrm{C} 5-\mathrm{C} 4-\mathrm{H} 4$ & 119.9 & $\mathrm{C} 13-\mathrm{N} 5-\mathrm{C} 15$ & $108.97(13)$ \\
\hline $\mathrm{C} 3-\mathrm{C} 4-\mathrm{H} 4$ & 119.9 & $\mathrm{C} 14-\mathrm{N} 5-\mathrm{C} 15$ & $108.22(14)$ \\
\hline $\mathrm{C} 6-\mathrm{C} 5-\mathrm{C} 4$ & $119.25(17)$ & $\mathrm{N} 5-\mathrm{C} 14-\mathrm{N} 3$ & $112.10(14)$ \\
\hline $\mathrm{C} 6-\mathrm{C} 5-\mathrm{H} 5$ & 120.4 & N5-C14-H14A & 109.2 \\
\hline $\mathrm{C} 4-\mathrm{C} 5-\mathrm{H} 5$ & 120.4 & $\mathrm{~N} 3-\mathrm{C} 14-\mathrm{H} 14 \mathrm{~A}$ & 109.2 \\
\hline $\mathrm{C} 7-\mathrm{C} 6-\mathrm{C} 5$ & $120.97(17)$ & N5-C14-H14B & 109.2 \\
\hline $\mathrm{C} 7-\mathrm{C} 6-\mathrm{O} 2$ & $118.98(16)$ & N3-C14-H14B & 109.2 \\
\hline $\mathrm{C} 5-\mathrm{C} 6-\mathrm{O} 2$ & $120.00(16)$ & $\mathrm{H} 14 \mathrm{~A}-\mathrm{C} 14-\mathrm{H} 14 \mathrm{~B}$ & 107.9 \\
\hline $\mathrm{C} 6-\mathrm{C} 7-\mathrm{C} 8$ & $119.33(17)$ & $\mathrm{N} 4-\mathrm{C} 15-\mathrm{N} 5$ & $111.96(14)$ \\
\hline $\mathrm{C} 6-\mathrm{C} 7-\mathrm{H} 7$ & 120.3 & $\mathrm{~N} 4-\mathrm{C} 15-\mathrm{H} 15 \mathrm{~A}$ & 109.2 \\
\hline $\mathrm{C} 8-\mathrm{C} 7-\mathrm{H} 7$ & 120.3 & $\mathrm{~N} 5-\mathrm{C} 15-\mathrm{H} 15 \mathrm{~A}$ & 109.2 \\
\hline $\mathrm{C} 3-\mathrm{C} 8-\mathrm{C} 7$ & $120.29(17)$ & $\mathrm{N} 4-\mathrm{C} 15-\mathrm{H} 15 \mathrm{~B}$ & 109.2 \\
\hline $\mathrm{C} 3-\mathrm{C} 8-\mathrm{H} 8$ & 119.9 & N5-C15-H15B & 109.2 \\
\hline $\mathrm{C} 7-\mathrm{C} 8-\mathrm{H} 8$ & 119.9 & $\mathrm{H} 15 \mathrm{~A}-\mathrm{C} 15-\mathrm{H} 15 \mathrm{~B}$ & 107.9 \\
\hline $\mathrm{C} 9-\mathrm{O} 2-\mathrm{C} 6$ & $116.62(13)$ & $\mathrm{C} 17-\mathrm{C} 16-\mathrm{H} 16 \mathrm{~A}$ & 109.5 \\
\hline $\mathrm{O} 3-\mathrm{C} 9-\mathrm{O} 2$ & $118.65(16)$ & $\mathrm{C} 17-\mathrm{C} 16-\mathrm{H} 16 \mathrm{~B}$ & 109.5 \\
\hline $\mathrm{O} 3-\mathrm{C} 9-\mathrm{B} 1$ & $130.19(16)$ & $\mathrm{H} 16 \mathrm{~A}-\mathrm{C} 16-\mathrm{H} 16 \mathrm{~B}$ & 109.5 \\
\hline $\mathrm{O} 2-\mathrm{C} 9-\mathrm{B} 1$ & $111.15(14)$ & $\mathrm{C} 17-\mathrm{C} 16-\mathrm{H} 16 \mathrm{C}$ & 109.5 \\
\hline $\mathrm{N} 2-\mathrm{B} 1-\mathrm{C} 9$ & $110.79(14)$ & $\mathrm{H} 16 \mathrm{~A}-\mathrm{C} 16-\mathrm{H} 16 \mathrm{C}$ & 109.5 \\
\hline $\mathrm{N} 2-\mathrm{B} 1-\mathrm{H} 1 \mathrm{~B} 1$ & 109.5 & $\mathrm{H} 16 \mathrm{~B}-\mathrm{C} 16-\mathrm{H} 16 \mathrm{C}$ & 109.5 \\
\hline $\mathrm{C} 9-\mathrm{B} 1-\mathrm{H} 1 \mathrm{~B} 1$ & 109.5 & $\mathrm{O} 5-\mathrm{C} 17-\mathrm{N} 6$ & $123.05(17)$ \\
\hline $\mathrm{N} 2-\mathrm{B} 1-\mathrm{H} 1 \mathrm{~B} 2$ & 109.5 & $\mathrm{O} 5-\mathrm{C} 17-\mathrm{C} 16$ & $120.52(17)$ \\
\hline $\mathrm{C} 9-\mathrm{B} 1-\mathrm{H} 1 \mathrm{~B} 2$ & 109.5 & $\mathrm{~N} 6-\mathrm{C} 17-\mathrm{C} 16$ & $116.43(16)$ \\
\hline
\end{tabular}




\begin{tabular}{|c|c|c|c|}
\hline $\mathrm{H} 1 \mathrm{~B} 1-\mathrm{B} 1-\mathrm{H} 1 \mathrm{~B} 2$ & 108.1 & $\mathrm{C} 17-\mathrm{N} 6-\mathrm{C} 18$ & $124.75(15)$ \\
\hline $\mathrm{C} 10-\mathrm{N} 2-\mathrm{C} 12$ & $107.66(13)$ & $\mathrm{C} 17-\mathrm{N} 6-\mathrm{H} 6 \mathrm{~N}$ & 117.6 \\
\hline $\mathrm{C} 10-\mathrm{N} 2-\mathrm{C} 13$ & $106.47(13)$ & $\mathrm{C} 18-\mathrm{N} 6-\mathrm{H} 6 \mathrm{~N}$ & 117.6 \\
\hline $\mathrm{C} 12-\mathrm{N} 2-\mathrm{C} 13$ & $107.04(13)$ & $\mathrm{C} 23-\mathrm{C} 18-\mathrm{C} 19$ & $119.95(17)$ \\
\hline $\mathrm{C} 10-\mathrm{N} 2-\mathrm{B} 1$ & $112.47(14)$ & $\mathrm{C} 23-\mathrm{C} 18-\mathrm{N} 6$ & $119.97(16)$ \\
\hline $\mathrm{C} 12-\mathrm{N} 2-\mathrm{B} 1$ & $113.27(13)$ & $\mathrm{C} 19-\mathrm{C} 18-\mathrm{N} 6$ & $119.95(16)$ \\
\hline $\mathrm{C} 13-\mathrm{N} 2-\mathrm{B} 1$ & $109.56(13)$ & $\mathrm{C} 20-\mathrm{C} 19-\mathrm{C} 18$ & $119.82(18)$ \\
\hline $\mathrm{N} 3-\mathrm{C} 10-\mathrm{N} 2$ & $111.84(14)$ & $\mathrm{C} 20-\mathrm{C} 19-\mathrm{H} 19$ & 120.1 \\
\hline $\mathrm{N} 3-\mathrm{C} 10-\mathrm{H} 10 \mathrm{~A}$ & 109.2 & $\mathrm{C} 18-\mathrm{C} 19-\mathrm{H} 19$ & 120.1 \\
\hline $\mathrm{N} 2-\mathrm{C} 10-\mathrm{H} 10 \mathrm{~A}$ & 109.2 & $\mathrm{C} 21-\mathrm{C} 20-\mathrm{C} 19$ & $119.93(17)$ \\
\hline $\mathrm{N} 3-\mathrm{C} 10-\mathrm{H} 10 \mathrm{~B}$ & 109.2 & $\mathrm{C} 21-\mathrm{C} 20-\mathrm{H} 20$ & 120.0 \\
\hline $\mathrm{N} 2-\mathrm{C} 10-\mathrm{H} 10 \mathrm{~B}$ & 109.2 & $\mathrm{C} 19-\mathrm{C} 20-\mathrm{H} 20$ & 120.0 \\
\hline $\mathrm{H} 10 \mathrm{~A}-\mathrm{C} 10-\mathrm{H} 10 \mathrm{~B}$ & 107.9 & $\mathrm{O} 4-\mathrm{C} 21-\mathrm{C} 20$ & $122.30(16)$ \\
\hline $\mathrm{C} 10-\mathrm{N} 3-\mathrm{C} 11$ & $108.32(13)$ & $\mathrm{O} 4-\mathrm{C} 21-\mathrm{C} 22$ & $117.09(16)$ \\
\hline $\mathrm{C} 10-\mathrm{N} 3-\mathrm{C} 14$ & $108.77(14)$ & $\mathrm{C} 20-\mathrm{C} 21-\mathrm{C} 22$ & $120.50(17)$ \\
\hline $\mathrm{C} 11-\mathrm{N} 3-\mathrm{C} 14$ & $108.20(14)$ & $\mathrm{C} 21-\mathrm{O} 4-\mathrm{H} 4 \mathrm{O}$ & 109.5 \\
\hline $\mathrm{N} 4-\mathrm{C} 11-\mathrm{N} 3$ & $112.61(14)$ & $\mathrm{C} 21-\mathrm{C} 22-\mathrm{C} 23$ & $119.29(17)$ \\
\hline N4-C11-H11A & 109.1 & $\mathrm{C} 21-\mathrm{C} 22-\mathrm{H} 22$ & 120.4 \\
\hline $\mathrm{N} 3-\mathrm{C} 11-\mathrm{H} 11 \mathrm{~A}$ & 109.1 & $\mathrm{C} 23-\mathrm{C} 22-\mathrm{H} 22$ & 120.4 \\
\hline N4-C11-H11B & 109.1 & $\mathrm{C} 18-\mathrm{C} 23-\mathrm{C} 22$ & $120.47(17)$ \\
\hline N3-C11-H11B & 109.1 & $\mathrm{C} 18-\mathrm{C} 23-\mathrm{H} 23$ & 119.8 \\
\hline $\mathrm{H} 11 \mathrm{~A}-\mathrm{C} 11-\mathrm{H} 11 \mathrm{~B}$ & 107.8 & $\mathrm{C} 22-\mathrm{C} 23-\mathrm{H} 23$ & 119.8 \\
\hline $\mathrm{O} 1-\mathrm{C} 2-\mathrm{N} 1-\mathrm{C} 3$ & $4.5(3)$ & $\mathrm{C} 11-\mathrm{N} 4-\mathrm{C} 12-\mathrm{N} 2$ & $-58.36(17)$ \\
\hline $\mathrm{C} 1-\mathrm{C} 2-\mathrm{N} 1-\mathrm{C} 3$ & $-176.59(16)$ & $\mathrm{C} 15-\mathrm{N} 4-\mathrm{C} 12-\mathrm{N} 2$ & $59.38(18)$ \\
\hline $\mathrm{C} 2-\mathrm{N} 1-\mathrm{C} 3-\mathrm{C} 8$ & $64.0(2)$ & $\mathrm{C} 10-\mathrm{N} 2-\mathrm{C} 12-\mathrm{N} 4$ & $56.54(17)$ \\
\hline $\mathrm{C} 2-\mathrm{N} 1-\mathrm{C} 3-\mathrm{C} 4$ & $-118.63(19)$ & $\mathrm{C} 13-\mathrm{N} 2-\mathrm{C} 12-\mathrm{N} 4$ & $-57.59(18)$ \\
\hline $\mathrm{C} 8-\mathrm{C} 3-\mathrm{C} 4-\mathrm{C} 5$ & $1.6(3)$ & $\mathrm{B} 1-\mathrm{N} 2-\mathrm{C} 12-\mathrm{N} 4$ & $-178.46(14)$ \\
\hline $\mathrm{N} 1-\mathrm{C} 3-\mathrm{C} 4-\mathrm{C} 5$ & $-175.82(15)$ & $\mathrm{C} 10-\mathrm{N} 2-\mathrm{C} 13-\mathrm{N} 5$ & $-57.80(17)$ \\
\hline $\mathrm{C} 3-\mathrm{C} 4-\mathrm{C} 5-\mathrm{C} 6$ & $-0.4(3)$ & $\mathrm{C} 12-\mathrm{N} 2-\mathrm{C} 13-\mathrm{N} 5$ & $57.13(18)$ \\
\hline $\mathrm{C} 4-\mathrm{C} 5-\mathrm{C} 6-\mathrm{C} 7$ & $-0.9(3)$ & $\mathrm{B} 1-\mathrm{N} 2-\mathrm{C} 13-\mathrm{N} 5$ & $-179.67(14)$ \\
\hline $\mathrm{C} 4-\mathrm{C} 5-\mathrm{C} 6-\mathrm{O} 2$ & $-178.39(15)$ & $\mathrm{N} 2-\mathrm{C} 13-\mathrm{N} 5-\mathrm{C} 14$ & $59.31(18)$ \\
\hline $\mathrm{C} 5-\mathrm{C} 6-\mathrm{C} 7-\mathrm{C} 8$ & $0.9(2)$ & $\mathrm{N} 2-\mathrm{C} 13-\mathrm{N} 5-\mathrm{C} 15$ & $-58.47(18)$ \\
\hline $\mathrm{O} 2-\mathrm{C} 6-\mathrm{C} 7-\mathrm{C} 8$ & $178.43(15)$ & $\mathrm{C} 13-\mathrm{N} 5-\mathrm{C} 14-\mathrm{N} 3$ & $-59.61(18)$ \\
\hline $\mathrm{C} 4-\mathrm{C} 3-\mathrm{C} 8-\mathrm{C} 7$ & $-1.5(3)$ & $\mathrm{C} 15-\mathrm{N} 5-\mathrm{C} 14-\mathrm{N} 3$ & $58.64(18)$ \\
\hline $\mathrm{N} 1-\mathrm{C} 3-\mathrm{C} 8-\mathrm{C} 7$ & $175.85(15)$ & $\mathrm{C} 10-\mathrm{N} 3-\mathrm{C} 14-\mathrm{N} 5$ & $59.51(19)$ \\
\hline $\mathrm{C} 6-\mathrm{C} 7-\mathrm{C} 8-\mathrm{C} 3$ & $0.3(3)$ & $\mathrm{C} 11-\mathrm{N} 3-\mathrm{C} 14-\mathrm{N} 5$ & $-57.96(18)$ \\
\hline $\mathrm{C} 7-\mathrm{C} 6-\mathrm{O} 2-\mathrm{C} 9$ & $105.80(17)$ & $\mathrm{C} 12-\mathrm{N} 4-\mathrm{C} 15-\mathrm{N} 5$ & $-59.85(19)$ \\
\hline $\mathrm{C} 5-\mathrm{C} 6-\mathrm{O} 2-\mathrm{C} 9$ & $-76.6(2)$ & $\mathrm{C} 11-\mathrm{N} 4-\mathrm{C} 15-\mathrm{N} 5$ & $57.99(18)$ \\
\hline $\mathrm{C} 6-\mathrm{O} 2-\mathrm{C} 9-\mathrm{O} 3$ & $-3.1(2)$ & $\mathrm{C} 13-\mathrm{N} 5-\mathrm{C} 15-\mathrm{N} 4$ & $59.57(19)$ \\
\hline $\mathrm{C} 6-\mathrm{O} 2-\mathrm{C} 9-\mathrm{B} 1$ & $176.90(15)$ & $\mathrm{C} 14-\mathrm{N} 5-\mathrm{C} 15-\mathrm{N} 4$ & $-58.56(18)$ \\
\hline $\mathrm{O} 3-\mathrm{C} 9-\mathrm{B} 1-\mathrm{N} 2$ & $17.3(3)$ & $\mathrm{O} 5-\mathrm{C} 17-\mathrm{N} 6-\mathrm{C} 18$ & $4.0(3)$ \\
\hline $\mathrm{O} 2-\mathrm{C} 9-\mathrm{B} 1-\mathrm{N} 2$ & $-162.61(14)$ & $\mathrm{C} 16-\mathrm{C} 17-\mathrm{N} 6-\mathrm{C} 18$ & $-176.28(16)$ \\
\hline $\mathrm{C} 9-\mathrm{B} 1-\mathrm{N} 2-\mathrm{C} 10$ & $56.76(19)$ & $\mathrm{C} 17-\mathrm{N} 6-\mathrm{C} 18-\mathrm{C} 23$ & $-130.07(19)$ \\
\hline $\mathrm{C} 9-\mathrm{B} 1-\mathrm{N} 2-\mathrm{C} 12$ & $-65.61(19)$ & $\mathrm{C} 17-\mathrm{N} 6-\mathrm{C} 18-\mathrm{C} 19$ & $54.1(2)$ \\
\hline $\mathrm{C} 9-\mathrm{B} 1-\mathrm{N} 2-\mathrm{C} 13$ & $174.96(14)$ & $\mathrm{C} 23-\mathrm{C} 18-\mathrm{C} 19-\mathrm{C} 20$ & $-0.5(3)$ \\
\hline $\mathrm{C} 12-\mathrm{N} 2-\mathrm{C} 10-\mathrm{N} 3$ & $-56.73(17)$ & $\mathrm{N} 6-\mathrm{C} 18-\mathrm{C} 19-\mathrm{C} 20$ & $175.33(16)$ \\
\hline $\mathrm{C} 13-\mathrm{N} 2-\mathrm{C} 10-\mathrm{N} 3$ & $57.78(17)$ & $\mathrm{C} 18-\mathrm{C} 19-\mathrm{C} 20-\mathrm{C} 21$ & $-1.1(3)$ \\
\hline
\end{tabular}




$\begin{array}{llll}\mathrm{B} 1-\mathrm{N} 2-\mathrm{C} 10-\mathrm{N} 3 & 177.79(13) & \mathrm{C} 19-\mathrm{C} 20-\mathrm{C} 21-\mathrm{O} 4 & -173.96(16) \\ \mathrm{N} 2-\mathrm{C} 10-\mathrm{N} 3-\mathrm{C} 11 & 58.28(17) & \mathrm{C} 19-\mathrm{C} 20-\mathrm{C} 21-\mathrm{C} 22 & 2.3(3) \\ \mathrm{N} 2-\mathrm{C} 10-\mathrm{N} 3-\mathrm{C} 14 & -59.11(17) & \mathrm{O} 4-\mathrm{C} 21-\mathrm{C} 22-\mathrm{C} 23 & 174.61(15) \\ \mathrm{C} 10-\mathrm{N} 3-\mathrm{C} 11-\mathrm{N} 4 & -60.21(18) & \mathrm{C} 20-\mathrm{C} 21-\mathrm{C} 22-\mathrm{C} 23 & -1.8(3) \\ \mathrm{C} 14-\mathrm{N} 3-\mathrm{C} 11-\mathrm{N} 4 & 57.55(18) & \mathrm{C} 19-\mathrm{C} 18-\mathrm{C} 23-\mathrm{C} 22 & 1.0(3) \\ \mathrm{N} 3-\mathrm{C} 11-\mathrm{N} 4-\mathrm{C} 12 & 60.24(18) & \mathrm{N} 6-\mathrm{C} 18-\mathrm{C} 23-\mathrm{C} 22 & -174.86(16) \\ \mathrm{N} 3-\mathrm{C} 11-\mathrm{N} 4-\mathrm{C} 15 & -57.68(18) & \mathrm{C} 21-\mathrm{C} 22-\mathrm{C} 23-\mathrm{C} 18 & 0.2(3)\end{array}$

Hydrogen-bond geometry $\left(A,{ }^{\circ}\right)$

\begin{tabular}{lllll}
\hline$D-\mathrm{H} \cdots A$ & $D-\mathrm{H}$ & $\mathrm{H} \cdots A$ & $D \cdots A$ & $D-\mathrm{H} \cdots A$ \\
\hline $\mathrm{C} 10-\mathrm{H} 10 A \cdots \mathrm{O} 3$ & 0.97 & 2.52 & $3.184(2)$ & 125 \\
$\mathrm{C} 12-\mathrm{H} 12 B \cdots \mathrm{O} 3$ & 0.97 & 2.46 & $3.135(2)$ & 126 \\
$\mathrm{~N} 1-\mathrm{H} 1 N \cdots{ }^{i}$ & 0.86 & 2.18 & $3.0217(19)$ & 168 \\
$\mathrm{O} 4-\mathrm{H} 4 O \cdots{ }^{i}{ }^{i i}$ & 0.82 & 1.85 & $2.6619(18)$ & 174 \\
$\mathrm{~N} 6-\mathrm{H} 6 N \cdots \mathrm{O} 1$ & 0.86 & 2.00 & $2.8415(19)$ & 166 \\
$\mathrm{C} 10-\mathrm{H} 10 B \cdots \mathrm{O} 3^{\mathrm{iii}}$ & 0.97 & 2.49 & $3.413(2)$ & 159 \\
$\mathrm{C} 15-\mathrm{H} 15 A \cdots \mathrm{O} 4^{\text {iv }}$ & 0.97 & 2.50 & $3.160(2)$ & 125 \\
$\mathrm{C} 20-\mathrm{H} 20 \cdots 5^{\mathrm{ii}}$ & 0.93 & 2.51 & $3.195(2)$ & 130
\end{tabular}

Symmetry codes: (i) $x,-y+3 / 2, z+1 / 2$; (ii) $x,-y+1 / 2, z-1 / 2$; (iii) $-x+1, y-1 / 2,-z+3 / 2$; (iv) $-x+1,-y+1,-z+1$. 